\title{
RESEARCH
}

Open Access

\section{Lack of additive role of ageing in nigrostriatal crossank neurodegeneration triggered by a-synuclein overexpression}

Mathieu Bourdenx ${ }^{1,2+}$, Sandra Dovero ${ }^{1,2+}$, Michel Engeln ${ }^{1,2}$, Simone Bido ${ }^{1,2}$, Matthieu F. Bastide ${ }^{1,2}$, Nathalie Dutheil ${ }^{1,2}$, Isabel Vollenweider ${ }^{3}$, Laetitia Baud ${ }^{3}$, Camille Piron ${ }^{1,2}$, Virginie Grouthier ${ }^{1,2}$, Thomas Boraud ${ }^{1,2}$, Grégory Porras ${ }^{4}$, Qin Li ${ }^{4,5}$, Veerle Baekelandt ${ }^{6}$, Dieter Scheller ${ }^{7}$, Anne Michel ${ }^{7}$, Pierre-Olivier Fernagut ${ }^{1,2}$, François Georges ${ }^{8,9}$, Grégoire Courtine ${ }^{3}$, Erwan Bezard ${ }^{1,2^{*}}$ and Benjamin Dehay ${ }^{1,2^{*}}$

\begin{abstract}
Introduction: Parkinson's disease (PD) is a progressive neurodegenerative disorder characterized by the loss of dopaminergic neurons as well as the presence of proteinaceous inclusions named Lewy bodies. a-synuclein (a-syn) is a major constituent of Lewy bodies, and the first disease-causing protein characterized in PD. Several a-syn-based animal models of PD have been developed to investigate the pathophysiology of PD, but none of them recapitulate the full picture of the disease. Ageing is the most compelling and major risk factor for developing PD but its impact on a-syn toxicity remains however unexplored. In this study, we developed and exploited a recombinant adeno-associated viral (AAV) vector of serotype 9 overexpressing mutated a-syn to elucidate the influence of ageing on the dynamics of PD-related neurodegeneration associated with a-syn pathology in different mammalian species.
\end{abstract}

Results: Identical AAV pseudotype 2/9 vectors carrying the DNA for human mutant p.A53T a-syn were injected into the substantia nigra to induce neurodegeneration and synucleinopathy in mice, rats and monkeys. Rats were used first to validate the ability of this serotype to replicate a-syn pathology and second to investigate the relationship between the kinetics of a-syn-induced nigrostriatal degeneration and the progressive onset of motor dysfunctions, strikingly reminiscent of the impairments observed in PD patients. In mice, AAV2/9-ha-syn injection into the substantia nigra was associated with accumulation of a-syn and phosphorylated ha-syn, regardless of mouse strain. However, phenotypic mutants with either accelerated senescence or resistance to senescence did not display differential susceptibility to ha-syn overexpression. Of note, p-a-syn levels correlated with nigrostriatal degeneration in mice. In monkeys, ha-syn-induced degeneration of the nigrostriatal pathway was not affected by the age of the animals. Unlike mice, $m$ onkeys did not exhibit correlations between levels of phosphorylated a-syn and neurodegeneration.

Conclusions: In conclusion, AAV2/9-mediated ha-syn induces robust nigrostriatal neurodegeneration in mice, rats and monkeys, allowing translational comparisons among species. Ageing, however, neither exacerbated nigrostriatal neurodegeneration nor a-syn pathology per se. Our unprecedented multi-species investigation thus favours the multiple-hit hypothesis for PD wherein ageing would merely be an aggravating, additive, factor superimposed upon an independent disease process.

Keywords: Ageing, a-synuclein, Animal models, Adeno-associated viral vector, Parkinson's disease

\footnotetext{
* Correspondence:

erwan.bezard@u-bordeaux.fr; benjamin.dehay@u-bordeaux.fr

${ }^{\dagger}$ Equal contributors

'University de Bordeaux, Institut des Maladies Neurodégénératives, UMR

5293, 33000 Bordeaux, France

Full list of author information is available at the end of the article
}

\section{() Biomed Central}

(c) 2015 Bourdenx et al. This is an Open Access article distributed under the terms of the Creative Commons Attribution License (http://creativecommons.org/licenses/by/4.0), which permits unrestricted use, distribution, and reproduction in any medium, provided the original work is properly credited. The Creative Commons Public Domain Dedication waiver (http:// creativecommons.org/publicdomain/zero/1.0/) applies to the data made available in this article, unless otherwise stated. 


\section{Introduction}

Parkinson's disease (PD) is characterized by a profound loss of dopaminergic neurons from the substantia nigra, pars compacta $(\mathrm{SNpc})$ associated with the presence of intraneuronal proteinaceous inclusions, named Lewy bodies (LB) in cell bodies and Lewy neurites (LN) in neuritis [1]. Although several genetic loci have been linked to the development of $\mathrm{PD}$, ageing remains the most prominent risk factor [2]. Two main hypotheses have been formulated regarding the influence of ageing: one proposes that ageing increases dopamine neuron susceptibility [3] and the other that PD arises from the combination of several insults over the individual's life [4]. Overall, the impact of ageing has been largely overlooked in preclinical studies of PD.

A major protein component of LB and $\mathrm{LN}$ is $\alpha$ synuclein ( $\alpha$-syn), a synaptic protein of, as yet, unclear function [5]. Interestingly, $\alpha$-syn has a unique importance in the aetiology of PD, as it appears to link familial and sporadic forms of the disease [6]. Several $\alpha$-syn transgenic mice have been developed, expressing human wild type (WT) or mutated $\alpha$-syn under different promoters [7]. They failed, however, to reproduce the combination of progressive and specific dopamine cell loss, protein aggregation and manifestation of parkinsonianlike symptoms [8]. Subsequently, the development of lentiviral and recombinant adeno-associated viral vectors (AAV) has opened up new possibilities regarding disease modelling. Nevertheless, the literature reports a bewildering variety of vectors, expression cassettes, serotypes, and titers, that hampers comparison between strains, species and investigators [8-10].

We, therefore investigated the spatial and temporal dynamics of dopamine neuron degeneration, human mutant p.A53T $\alpha$-syn (ho-syn) expression and the resulting synucleinopathy using AAV pseudotype 2/9 (AAV2/9) in different species, namely mouse, rat and marmoset monkey. After replicating the progressive degeneration in rats and characterizing their parkinsonian-like phenotype, we questioned whether ageing influences susceptibility of dopamine neurons to neurodegeneration using senescence-prone and -resistant mouse strains and a population of marmoset monkeys featuring young and aged individuals. Our results demonstrate that ageing does not amplify the neurodegenerative process but simply adds to the $\alpha$-syn overexpression supporting the multi-hit hypothesis.

\section{Materials and methods}

\section{Adeno-Associated Viral vector production}

Recombinant AAV2/9-p.A53T-human- $\alpha$-syn (AAV-h $\alpha-$ syn) vectors were produced by polyethylenimine (PEI) mediated triple transfection of low passage HEK-293 T /17 cells (ATCC; cat number CRL-11268). The AAV expression plasmid pAAV2-CMVie/hSyn-synA53TWPRE-pA (provided by Dr V. Baekelandt) was cotransfected with the adeno helper pAd Delta F6 plasmid (Penn Vector Core, cat \# PL-F-PVADF6) and AAV Rep Cap pAAV2/9 plasmid (Penn Vector Core, cat \# PL-TPV008).

AAV vectors were purified as previously described [11]. Cells are harvested $72 \mathrm{~h}$ post transfection, resuspended in lysis buffer $(150 \mathrm{mM} \mathrm{NaCl}, 50 \mathrm{mM}$ Tris- $\mathrm{HCl}$ $\mathrm{pH}$ 8.5) and lysed by 3 freeze-thaw cycles $\left(37^{\circ} \mathrm{C} /-80^{\circ} \mathrm{C}\right)$. The cell lysate is treated with 150 units $/ \mathrm{ml}$ Benzonase (Sigma, St Louis, MO) for $1 \mathrm{~h}$ at $37{ }^{\circ} \mathrm{C}$ and the crude lysate is clarified by centrifugation. Vectors are purified by iodixanol step gradient centrifugation, and concentrated and buffer-exchanged into Lactated Ringer's solution (Baxter, Deerfield, IL) using vivaspin20 $100 \mathrm{kDa}$ cut off concentrator (Sartorius Stedim, Goettingen, Germany).

Titrations were performed at the transcriptome core facility (Neurocentre Magendie, INSERM U862, Bordeaux, France). The genome-containing particle (gcp) titer was determined by quantitative real-time PCR using the Light Cycler 480 SYBR green master mix (Roche, cat \# 04887352001) with primers specific for the AAV2 ITRs (fwd 5'-GGAACCCCTAGTGATG GAGTT-3'; rev 5'-CGGCCTCAGTGAGCGA-3') [12] on a Light Cycler 480 instrument.

Purity assessment of vector stocks was estimated by loading $10 \mu \mathrm{l}$ of vector stock on $10 \%$ SDS acrylamide gels, total proteins were visualized using the Krypton Infrared Protein Stain according to the manufacturer's instructions (Life Technologies).

\section{Animals}

Experiments were performed in accordance with the European Union directive of September 22, 2010 (2010/ $63 / \mathrm{EU}$ ) on the protection of animals used for scientific purposes. The Institutional Animal Care and Use Committee of Bordeaux (CE50) approved experiments under the license number 5012099-A (rodents) and 50120102A (monkeys). Kinematics experiments were performed under the guidelines established at EPFL. Local Swiss Veterinary Offices approved all the procedures. Experiments were performed on Sprague Dawley (53 animals), Wistar (8 animals) and Lewis rats (10 animals) with initial weight of approximately $200 \mathrm{~g}$ and age of 8 weeks. Rats were ordered from Janvier Labs or Charles River in France. Mouse experiments were performed on C57Bl/ 6 J (12 animals - ordered from Charles River laboratories), SAMR1 (19 animals) and SAMP8 (21 animals). Both SAMR1 and SAMP8 were purchased from the research animal facility of the Barcelona Science Park (Barcelona, Spain). 
Monkey experiments were performed in an Association for Assessment and Accreditation of Laboratory Animal Care accredited facility following acceptance of study design by the Institute of Lab Animal Science (Chinese Academy of Science, Beijing, China) Institutional Animal Care and Use Committee. Thirteen male marmoset monkeys (Callithrix jacchus; Beijing, People's Republic of China) were housed in 2 primate cages, allowing visual contacts and interactions with monkeys housed inside the cage. The use of wood chip or shredded paper litter on the cage floor is a source of environmental enrichment. Food and water were available ad libitum and animal care was supervised daily by veterinarians skilled in the health care and maintenance of nonhuman primates.

\section{Rodent experiments}

\section{1-Methyl-4-phenyl-1,2,3,6-tetrahydropyridine-intoxicated} mice

Eight- to 10-week-old male C57BL/6 J, SAMP8 and SAMR1 mice received one intraperitoneal injection of 1methyl-4-phenyl-1,2,3,6-tetrahydropyridine (MPTP)- $\mathrm{HCl}$ per day (30 mg/kg free base; Sigma) for five consecutive days. Control mice received saline injections only. Mice were killed five months after MPTP intoxication. Four to nine mice were used in each group.

\section{Surgical procedures}

All the interventions were performed under full general anesthesia with isoflurane in oxygen-enriched air (1$2 \%)$. After surgery, rodents were placed in an incubator for optimized recovery from anesthesia. Rats were injected either bilaterally (kinematic recordings,) or unilaterally (immunohistochemical investigation and basic motor behavior (spontaneous locomotor activity and stepping test)) in the SNpc with the AAV-ho-syn $(2 \mu \mathrm{l}-$ $\left.7.0 \times 10^{12} \mathrm{vg} / \mathrm{ml}\right)$ or the control AAV-GFP $\left(7.0 \times 10^{12}\right.$ $\mathrm{vg} / \mathrm{ml}$ - solely for kinematic recordings). Under isoflurane anesthesia, rats were placed in a stereotaxic frame (Kopf Instruments) and received two bilateral intranigral injections (Anteroposterior: -4.9 and -5.4 ; Mediolateral: \pm 2.2 and \pm 2 ; Dorsoventral: -7.8 , in $\mathrm{mm}$ from bregma) of either vector, as previously described [13]. Mice were injected unilaterally with the AAV-ho-syn $\left(120 \mathrm{nl}-7.0 \times 10^{12} \mathrm{vg} / \mathrm{ml}\right)$ in the right SNpc (coordinates from Bregma: $\mathrm{AP}=-2.9, \mathrm{~L}=-1.3$, DV $=-4.5)$. Viruses were injected with a glass pipette at $0.4 \mu \mathrm{l} \cdot \mathrm{min}^{-1}$ and the pipette was left in place for $4 \mathrm{~min}$ after injection to avoid leakage.

\section{Kinematic Recordings}

All procedures used have been detailed previously [14]. Briefly, whole-body kinematics were recorded using the high-speed motion capture system Vicon (Vicon Motion
Systems, UK), combining 12 infrared cameras (200 Hz). $4 \mathrm{~mm}$ reflective markers were attached bilaterally overlying the iliac crest, the greater trochanter (hip), the lateral condyle (knee), the malleolus (ankle), and the base of the metatarsal phalageal joint (MTP). 3D position of the markers was reconstructed offline using Vicon Nexus software (1.8). The body was modeled as an interconnected chain of rigid segments, and joint angles were generated accordingly. For subsequent kinematic analysis, only hindlimb and parameters related to the trunk were analyzed. Parameters (Additional file 1: Table S1) describing gait timing, joint kinematics, limb endpoint trajectory, and trunk stability were computed for each gait cycle using custom written MATLAB scripts and according to methods described previously [14].

\section{Behavioral Tasks}

Spontaneous locomotor actimetry Spontaneous locomotor activity was evaluated in cages similar to home cages $(35 \times 25 \times 25 \mathrm{~cm})$ flanked with photobeam to allow computerized counting of horizontal beam breaks (protocol adapted from [15]. After a $3 \mathrm{~h}$ habituation session, each subsequent recording sessions (i.e., Bsl, $4,8,12,16$ weeks) lasted $3 \mathrm{~h}$.

Stepping test Forelimb akinesia was assessed using stepping [13, 16]. Briefly, animals were gently held and conducted over a $90 \mathrm{~cm}$ distance to allow forehand followed by backhand steps count. Left and right limb performances were successively evaluated over 2 daily sessions on 3 consecutive days. The average number of left/right forehand steps was averaged over the 6 sessions for each time-point.

Kinematic recordings Rats were handled and trained during one week prior to surgery in order to accustom them to the two locomotor tasks. After AAV-h $\alpha$-syn injection surgery animals were trained 2-3 times per week and monitored visually to assess whether PD-like symptoms had developed. Out of 8 rats initially injected 6 displayed observable bradykinesia and were thus included in the functional analysis. Symptoms emerged at different timepoints for the individual rats (8-16 weeks post injection) and the recordings used for analysis are from these chronic timepoints. Due to improved functional performance with training animals were only trained once a week after symptoms had emerged. Overground and ladder locomotion were recorded on different days. One rat was not included in the ladder analysis since not enough functional steps could be recorded from this animal in this task. AAV-h $\alpha$-syn rats were compared to a set of healthy rats that were extensively trained to perform both locomotor tasks. 
Overground locomotion was tested on a $15 \mathrm{~cm}$ wide and $120 \mathrm{~cm}$ long, elevated runway. 10-15 steps per side were analyzed per rat. A total of 89 kinematic parameters were computed and included in the subsequent PC analysis for this task (Additional file 1: Table S1).

Crossing of an elevated horizontal ladder (rung spacing: $5.2+/-0.3 \mathrm{~cm}$ ) was tested in alternation with overground walking. 10-15 steps per side were analyzed per rat. Quantification of hit, slip or miss paw placement was assessed from slow motion videos acquired at $100 \mathrm{~Hz}$.

\section{Principal Component Analysis}

Behavioral data was analyzed by PC analysis [17]. Not all the steps are depicted in the figures.

Step 1: Continuous locomotion is recorded using a high-resolution kinematic system.

Step 2: Custom-written MATLAB scripts are applied to reconstructed kinematic data in order to compute basic parameters and highly elaborated variables that provide a comprehensive quantification of gait features. All variables computed are specified in Additional file 1: Table S1. Approximately 10-15 steps were extracted per rat and experimental condition.

Step 3: The matrix combining all values of variables from all rats and steps was then subjected to a PC analysis. For this purpose, we used the correlation method, which adjusts the mean of the data to 0 and the SD to 1 . This method allows the comparison of variables with disparate values (large versus small), and/or different variances. The result of the PC analysis is a new set of synthetic uncorrelated variables, i.e., the PCs, which each explains the maximum possible amount of variance.

Step 4: The new coordinates of gait patterns along each PC, termed PC scores, are extracted for each rat. PC scores are used to represent gait patterns in the "denoised" PC space to visualize differences between mice and experimental conditions.

Step 5: PC scores are averaged for each experimental condition and represented in histogram plots to identify the type of information differentiated along each $\mathrm{PC}$ axis.

Step 6: Each PC is a linear combination of the original parameters with appropriate weights, which are termed "factor loadings." The values of factor loadings range from -1 to 1 , and correspond to correlations between original parameters and a given PC.

Step 7: Factor loadings with a high value (|factor loading $\mid>0.5$ ) are extracted, color-coded based on their correlation value, and regrouped into functional clusters based on the type of gait control aspects they refer to. This process leads to an objective extraction of the most relevant behavioral parameters to account for the effects of a specific experimental condition (Additional file 2: Figure S6).

Step 8: To provide a more classical representation of the observed effects, relevant parameters representative of functional clusters are extracted and represented in histogram plots.

\section{Primate experiments \\ Surgery}

Eight two-years-old (young group) and five six-years-old (old group) common marmosets (Callithrix jacchus) were unilaterally injected with $4 \mu \mathrm{L}$ AAV-ho-syn in 2 points of the SNpc following electrophysiological recordings (AP: +4 and $+3.3 \mathrm{~mm}, \mathrm{~L}:+3.7$ and $+3.5 \mathrm{~mm}$, $\mathrm{D}:+15 \mathrm{~mm}$ from interaural line). Recording system: 16 channel wireless system, Multichannel Systems, Reutlingen, BW, Germany). Animals were anaesthetized with atropine $\mathrm{SO}_{4}$ at $0.04 \mathrm{mg} / \mathrm{kg}$, i.m. prior to preparation for surgery. At least $10 \mathrm{~min}$ later, the animals were anaesthetized with ketamine $\mathrm{HCl}$ at $10 \mathrm{mg} / \mathrm{kg}$, IM. Following stereotaxic injections, viral expression was allowed for the next eleven weeks.

\section{Post-mortem processing}

At the end of different experiments, animals were killed by sodium pentobarbital overdose $(150 \mathrm{mg} / \mathrm{kg}$, i.v.), and perfused transcardiacally with $0.9 \%$ saline solution (containing $1 \%$ heparin) followed by $4 \%$ PFA performed in accordance with accepted European Veterinary Medical Association guidelines. Brains were removed quickly after death and post-fixed overnight in the same fixative, then cryoprotected in PBS containing $20 \%$ sucrose and frozen by immersion in a cold isopentane bath $\left(-45^{\circ} \mathrm{C}\right)$, before being stored at $-80{ }^{\circ} \mathrm{C}$ until sectioning. Brains were sectioned in a Leica CM3050S cryostat (Leica Microsystems, Wetzlar, Germany) at $-20{ }^{\circ} \mathrm{C}$. Brains were cut at $50 \mu \mathrm{m}$-thick sections and both striatal and SNpc levels were collected.

\section{Immunohistochemistry}

Extent of lesion To assess the integrity of the nigrostriatal pathway, tyrosine hydroxylase $(\mathrm{TH})$ immunohistochemistry was performed on striatal and SN free-floating sections (Additional file 3: Table S2). On the rat experiment, dopamine transporter (DAT) immunochemistry on striatal sections was also done. Briefly, striatal sections from three representative levels (anterior, medial and posterior) were incubated with a mouse monoclonal antibody raised against human TH (Millipore, MAB318, $1 / 5000)$ or rabbit polyclonal antibody raised against 
DAT ([18], 1/5 000) for $72 \mathrm{~h}$ at room temperature. The staining was revealed by a specific peroxidase EnVision ${ }^{\mathrm{mm}}$ system (mouse or rabbit HRP EnVison ${ }^{\text {TM }}$ kit DAB+ DAKO, K4007 or K4011) followed by DAB visualization. Midbrain sections containing the SNpc were processed for tyrosine hydroxylase. Serial Free-floating sections were incubated in mouse monoclonal $\mathrm{TH}$ antibody (Millipore, MAB318, 1/5 000) for one night at room temperature and revealed by an anti-mouse peroxidase EnVision $^{\text {TM }}$ system followed by DAB staining. SN free floating sections are mounted on gelatinized slides, counterstain with $0.1 \%$ cresyl violet solution, dehydrated and coversliped while striatal sections are mounted on gelatinized slides and coversliped only.

Human $\boldsymbol{\alpha}$-syn expression Human $\alpha$-syn expression levels were revealed in the striatum (anterior, median and posterior part) and in the SNpc by immunohistochemistry (Additional file 3: Table S2). The selected sections of the striatum, and serial free-floating sections of the SN were incubated with a mouse monoclonal antibody raised against human $\alpha$-syn (clone syn211 Thermo Scientific, MS1572, 1:1000) for one night at room temperature and revealed by an anti-

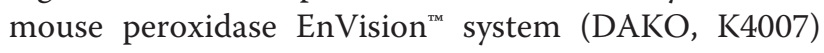
followed by DAB incubation. Phosphorylated synuclein immunochemistry was investigated in the substantia nigra in mouse and marmoset experiments. Briefly, serial sections of the SN were incubated for 3 nights at room temperature in a rabbit anti $\mathrm{p}$-Syn (Abcam AB51253 - 1/500) and revealed with the rabbit HRP EnVision polymere system $^{\mathrm{Tm}}$ followed by $\mathrm{DAB}$ as substrate. Free-floating sections were mounted on gelatinized slides, dehydrated and coverslipped before further analysis.

Fluorescence co-localization Co-localization of Ubiquitin and TH with $\alpha$-syn was revealed by a triple fluorescence immunohistochemistry within the SNpc of sham or AAV-ho-syn injected rat at 16 week post-surgery. All sections were individually identified to perform staining with all sections in the same conditions. After a blocking step in $5 \%$ fat milk, TH was first revealed with a sheep anti TH (Abcam -1/1000) for one night followed by a donkey anti-sheep Alexa 488 (1/500-1 h). The staining was briefly observed under microscope before to continue with a simultaneous incubation of a mouse anti- $\alpha-$ syn and rabbit anti-Ubiquitin both diluted at 1/1000 in PBS. Then, the $\alpha$-syn signal is revealed first by incubate sections in a Alexa Cys5 goat anti mouse for $1 \mathrm{~h}$ at RT and the double staining TH- $\alpha$-syn was observed before the last staining with an anti-rabbit HRP EnVision ${ }^{\mathrm{TM}}$ system (DAKO, K4011) followed by an 549 Dylight anti HRP diluted at $1 / 1000$ for $1 \mathrm{~h}$. Free-floating sections were mounted on slides and coverslipped with fluoromount $^{\mathrm{Tm}}$ aqueous mounting media (Sigma, F4680).

\section{Image analysis}

Grey level TH quantification in the striatum TH level in the striatum is quantified by densitometry. Sections are scanned in an Epson expression 10000XL high-resolution scanner and images are used in ImageJ software to compare the grey level in the delineated striatum for each animal.

\section{Stereological assessment of TH positive cells in the} SN TH-positive SN cells were counted by stereology blind with regard to the experimental condition using a Leica DM6000B motorized microscope coupled with the Mercator Pro software (ExploraNova, La Rochelle, France).The SN was delineated for each section and probes for stereological counting were applied to the map obtained (sampling and size of probes, see Additional file 4: Table S3). Each TH-positive cell with its nucleus included in the probe or intersecting any of the acceptance lines was counted. The optical fractionator method was finally used to estimate the total number of $\mathrm{TH}$-positive cells in the SN of each animal.

$\alpha$-syn and DAT surface quantification in the STR $\alpha$ syn expression and DAT staining were quantified in the striatum on high-resolution images from NanoZoomer 2.0 HT (BIC facility). Each NDPI image obtained was used in Mercator Pro software (Explora Nova, La Rochelle, France) to quantify the representative surface of $\alpha$-syn or DAT staining within the striatum. Briefly, striata were delineated and a color threshold was applied for each image using the same parameters allowing comparison between experimental groups. The surface detected was then compared to the whole striatum surface in order to obtain a percentage of stained structure.

\section{$\alpha$-syn and p- $\alpha$-syn volume quantification in the $S N$} In order to quantify the expression of $\alpha$-syn or $p$ - $\alpha$-syn in the whole $\mathrm{SN}$, a semi-stereological assessment was used for each animal. As for the striatal $\alpha$-syn quantification, all slides with serial sections of the whole SNpc were scanned in a NanoZoomer 2.0 HT (BIC facility). First, NDPI images obtained were analyzed in Mercator Pro software (Explora Nova, La Rochelle, France) to quantify the representative surface of the staining in each SN section using a color threshold. Then, surfaces detected were reported to the sampling scheme (Additional file 4: Table S3) to assess the representative volume of $\alpha$-syn or $p$ - $\alpha$-syn expression for each SN using the Cavalieri method. Finally the percentage of SN 
volume stained for $\alpha$-syn was used to compare the effect of each treatment.

\section{Results}

a-syn overexpression induces a progressive degeneration of the nigrostriatal pathway in rat

To determine the course of dopamine neuron degeneration, adult Sprague-Dawley rats received intranigral stereotactic injection of AAV2/9 carrying human p.A53T $\alpha$-syn (AAV-ho-syn). We then assessed several markers of the nigrostriatal pathway: tyrosine hydroxylase $(\mathrm{TH})$ and dopamine transporter (DAT) immunoreactivities in the striatum as well as the number of TH-positive cells in the SNpc which all correlated with the protein expression of ha-syn in both striatum and SNpc at five time points $(72 \mathrm{~h}, 1$, 4,8 and 16 weeks) (Fig. 1). Intranigral injection of AAV-h $\alpha$-syn resulted in a markedly significant reduction of dopaminergic fibers, evidenced by a reduction of TH (Fig. 1a and f, Additional file 5: Figure S1a) and DAT (Fig. 1b and f, Additional file 5: Figure S1b) immunostaining, beginning at 1 week and reaching a plateau at 4 weeks, associated with progressive dopamine neuron death ranging from $50 \%$ at 1 week to approximately $80 \%$ at 16 weeks after surgery (Fig. 1d and $\mathrm{f}$, Additional file 5: Figure S1c). A two-phase exponential decay equation models the time-course of $\mathrm{SNpc}$ dopaminergic neuron loss $(y=2230+$ $3707.2728 \mathrm{e}^{-2.171 \mathrm{x}}+3417.877 \mathrm{e}^{-0.1069 \mathrm{x}}, r^{2}=0.66$, Fig. 1f). These results indicate that nigral expression of ho-syn induces progressive dopamine neuron degeneration in rat, in agreement with previous findings $[10,19,20]$.

We next attempted to examine the ho-syn expression pattern in SNpc and striatum over time. Coinciding with the onset of neurodegeneration ( $72 \mathrm{~h}$ after injection), we observed a progressive increase in h $\alpha$-syn around the injection site at $72 \mathrm{~h}$ that peaked at 8 weeks post-injection (Fig. 1e and f, Additional file 5: Figure S1d). In the striatum, h $\alpha$-syn staining was detected weakly at 1 week and reached its highest level at 4 weeks (Fig. 1c and f, Additional file 5: Figure S1e) concomitantly with the significant decrease in striatal dopamine markers (Additional file 5: Figure S1a and b).

Anatomically, expression of ho-syn started in the SNpc before being noticeable in the striatum (Additional file 6: Figure S2a and b). A strong intraneuronal ho-syn staining was observed at $72 \mathrm{~h}$ that then changed to a diffuse pattern in later time points (Additional file 6: Figure S2a). The striatum, on the other hand, showed long, wire-like, staining from 1 week onwards, subsequently changing to a more punctate form (Additional file 6: Figure S2b). In the $\mathrm{SNpc}, \mathrm{h} \alpha$-syn co-localized with ubiquitin in neuronal cells at 8 weeks after injection (Additional file 7: Figure S3e-1), in contrast to sham-operated rats (Additional file 7: Figure
S3a-d). Staining of ha-syn phosphorylated at S129 (p- $\alpha-$ syn), a common marker for pathological $\alpha$-syn in postmortem tissue [21], was observed specifically within SNpc neurons in AAV-ho-syn-injected rats at 16 weeks post-injection (Additional file 8: Figure S4b-e) but not in sham-operated rats (Additional file 8: Figure S4a). P- $\alpha$-syn staining was mostly observed in perikarya of neuronal cells as well as in dendritic terminals (Additional file 8: Figure S4c-d). While ho-syn staining spread throughout the whole mesencephalon at 16 weeks after surgery (Fig. 1e), p- $\alpha$-syn immunolabelling remained restricted to SNpc neurons (Additional file 8: Figure S4). These results indicate the presence of intracytoplasmic accumulation of $\alpha$-syn, positive either for ubiquitin or for $\mathrm{p}-\alpha$-syn, in AAVho-syn-injected rats.

Because earlier work has shown differential rodent strain neurodegeneration susceptibility to neurotoxin [22], we then investigated whether two common laboratory rat strains, i.e., Sprague-Dawley and Wistar, would display a different susceptibility to h $\alpha$-syn overexpression. Strikingly, Wistar rats were significantly less susceptible to dopamine neuron degeneration (45\% at 8 weeks) compared to Sprague-Dawley rats (69 \% at 8 weeks) (Additional file 9: Figure S5), highlighting the importance of the genetic background in the response to ho-syn overexpression.

\section{a-syn overexpression induces a progressive motor dysfunction in rats}

The extent of lesion observed suggested that AAVho-syn-injected rats might develop overt parkinsonism. While AAV-ho-syn-injected rats displayed a significant decrease in spontaneous activity (Fig. 2a) from 8 weeks post-surgery onwards, they showed a significant decrease in the number of adjusting steps as early as 4 weeks and thereafter (Fig. 2b).

We then conducted high-resolution kinematic analyses to obtain quantified and detailed analyses of motor impairments. We first evaluated the rats during selfinitiated overground locomotion. While sham rats fluidly crossed the runway at a fast pace, AAV-h $\alpha$-syn-injected rats walked slower and exhibited a more variable and shuffling gait pattern (Fig. 2c). To measure these deficits, we computed 89 parameters providing quantitative measurements of bilateral gait features (Additional file 1: Table S1), and submitted all the parameters to a principal component (PC) analysis ( $n=4$ sham; $n=6 \mathrm{AAV}-\mathrm{h} \alpha$ syn rats; $10-15$ steps/rat/side). This method allows the objective identification of the specific features that are affected or not affected by injuries or experimental conditions [23]. Representation of all data points in the denoised space created by PC1-3 (Fig. 2d) showed that the gait patterns of healthy and AAV-h $\alpha$-syn-injected rats clustered in distinct spaces that minimally overlapped. 

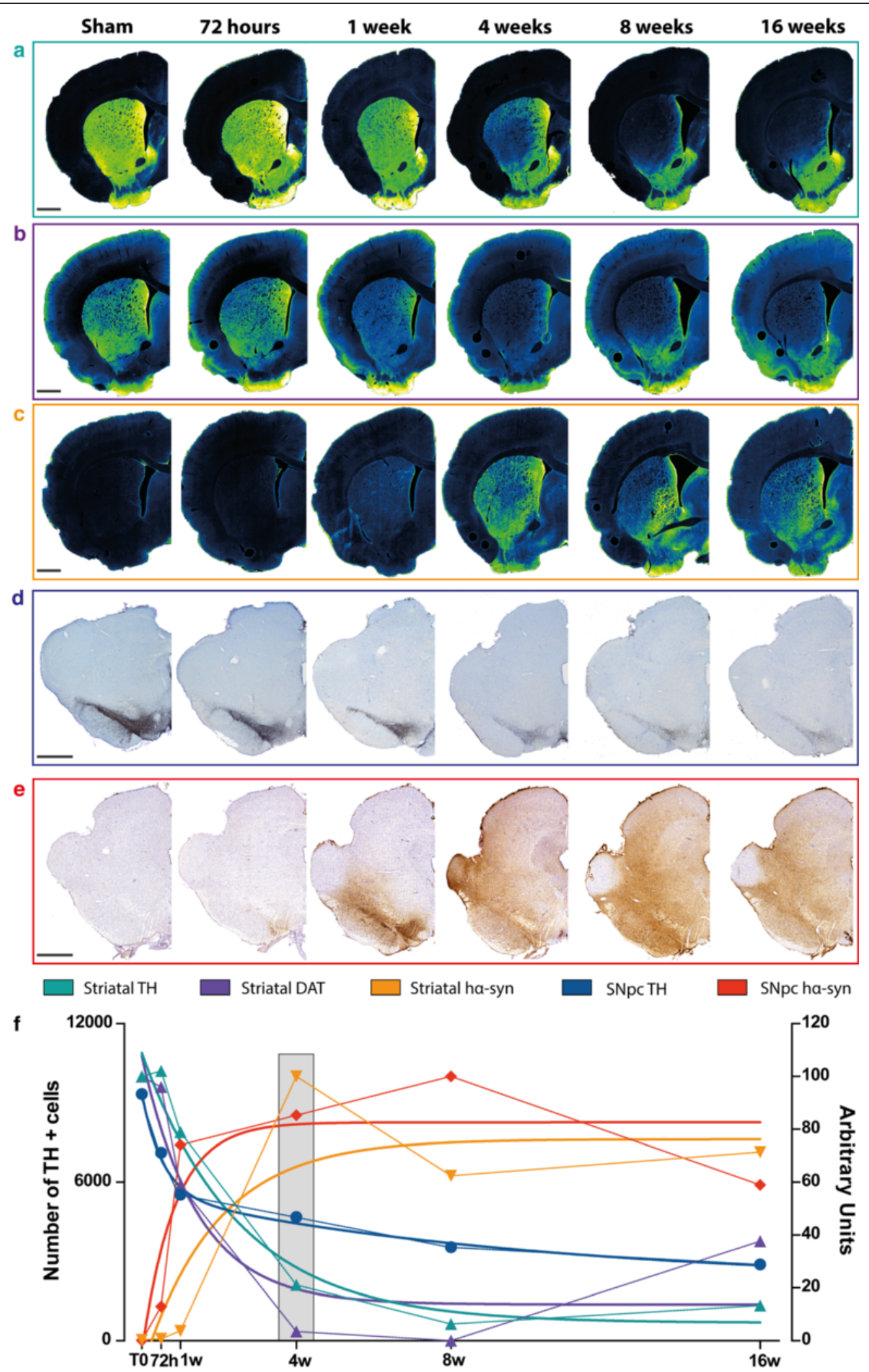

Fig. 1 (See legend on next page.) 
(See figure on previous page.)

Fig. 1 rAAV2/9 vector-mediated overexpression of ha-syn in rat SNpc induces progressive dopaminergic neurodegeneration related to ha-syn expression dynamics. (a-e) Representative photomicrographs of dopaminergic markers and human a-syn immunostaining at striatal (a-c) and SNpc (d,e) levels at several time points after injection. (a,d): tyrosine hydroxylase (TH); (b): dopamine transporter (DAT); (c,e): human a-syn. (f) Quantification of the different markers over time. Briefly, stereological quantification of the number of TH-positive cells in the SNpc of rat are reported on the left axis, all staining intensity have been normalized between min (0 \%) and max (100 \%) and reported on the right axis to allow comparison of the evolution. Dot-lines represent actual values while plain curves are regression curves. Colors are the same as in the upper part of the panel: light blue: striatal TH, purple: striatal DAT, yellow: striatal a-syn, blue: SNpc TH, red: SNpc a-syn. Scales applies to all pictures: 1 mm

Analysis of individual scores on PC1, which explained $18 \%$ of the total variance, revealed a significant difference between healthy and AAV-h $\alpha$-syn-injected rats $(p<0.01$; Fig. 2d). Thus, PC1 distinguished healthy from AAV-h $\alpha$-syn-injected rats. We next extracted the parameters correlated with the AAV-h $\alpha$-syn-specific PC1 (|factor loading $\mid>0.5$ ), and regrouped them in functional clusters (Additional file 2: Figure S6). $38 \%$ of all parameters analysed were altered in AAV-ho-syn-injected rats compared to healthy animals. Analyses of these parameters revealed that AAV-ho-syn-injected rats displayed altered gait timing, reduced control over limb endpoint trajectory and velocity, increase medio-lateral instability and aberrant inter-limb coordination (Fig. 2e).

We then tested rats during walking on a horizontal ladder, requiring precision in foot placement. Whereas sham rats progressed across the ladder with ease, AAV-ho-syn-injected rats crossed the ladder at a reduced velocity, with occasional periods of freezing (Additional file 10: Movie S1). They exhibited inaccuracy in the positioning of the hind paws onto the rungs compared to healthy rats (Fig. 2f). This misplacement resulted in an increased percentage of slips (healthy: 7.4 +/- $0.3 \%$; AAV-h $\alpha$-syn rats: $13.1+/-$ $4.7 \%$ ) and misses (healthy: $1.2+/-1 \%$; AAV-h $\alpha$-syn: $7.6+/-4.8 \%$ ) in AAV-ho-syn-injected rats (Fig. 2g). While individual rats displayed variable behaviours on the ladder (Fig. 2g), this precision task uncovered more pronounced motor deficits compared to basic runway locomotion.

Taken together, these data indicate that the ho-syninduced nigrostriatal degeneration in the rat is accompanied by a progressive onset of motor dysfunctions reminiscent of the impairments observed in PD patients.

\section{Premature ageing mice did not present higher susceptibility to a-syn overexpression}

Because ageing remains the most compelling risk factor for PD [2], we next investigated the relation between ageing and ho-syn-induced neurodegeneration using a panel of mice strains. The $\mathrm{C} 57 \mathrm{Bl} / 6 \mathrm{~J}$, the senescence-accelerated mouse-prone 8 (SAMP8), the senescence-accelerated mouse-resistant 1 (SAMR1), and their littermate controls were used. The SAMP8 mice are naturally phenotypic mutant born from a collection of inbred mice that exhibit ageing alterations, such as age-dependent early onset of senile amyloidosis, degenerative arthropathy, cataracts, osteoporosis and osteoarthritis, reduced fecundity, early loss of fecundity and a shorter life span (death typically occurs at 8 months) [24]. The SAMR1 mice come from a collection of three long-lived control strains [24]. SAMR1 and SAMP8 mice were raised on an AKR/J genetic background.

The integrity of the nigrostriatal pathway was assessed at 20 weeks after stereotactic surgery to cover the life span of SAMP8 mice. While all strains displayed a significant decrease in striatal $\mathrm{TH}$ immunoreactivity, only C57Bl/6 J mice presented a significant decrease in the number of TH-positive neurons in the SNpc (Fig. 3a). SAMP8 and SAMR1 mice, on the AKR/J background, are less susceptible to h $\alpha$-syn-induced degeneration than $\mathrm{C} 57 \mathrm{Bl} / 6 \mathrm{~J}$ mice. Whether this difference actually represents a delayed degeneration or an absence of degeneration remains to be fully established.

We then examined the occurrence and pattern of ho-syn pathology at both striatal and nigral levels. All strains demonstrated strong ha-syn staining at both levels (Fig. 3b). In contrast to rats, $\mathrm{p}-\alpha$-syn staining was not restricted to SNpc (Fig. 3c). Further examination revealed an intriguing pattern for $\mathrm{p}-\alpha$-syn staining: $\mathrm{C} 57 \mathrm{Bl} / 6 \mathrm{~J}$ exhibited the strongest pattern and SAMP8 the weakest (Fig. 3c). Interestingly, $\mathrm{p}-\alpha$-syn levels and the number of TH-positive cells in the SNpc were covariant: the strain with the highest amount of $\mathrm{p}$ - $\alpha$-syn (i.e., $\mathrm{C} 57 \mathrm{Bl} / 6 \mathrm{~J}$ ) was also the one that displayed the highest dopamine neuron death (Fig. 3d).

To establish that SAMP8 and SAMR1 mice were less susceptible to PD-related neurodegeneration, animals were treated with a subacute regimen of intoxication with 1-methyl-1,2,3,6-tetrahydropyridine (MPTP), a proparkinsonian mitochondrial neurotoxin that reproduces several aspects of PD [25]. Only C57Bl/6 J mice displayed significant DA neurodegeneration 5 months following MPTP intoxication, a result reminiscent of our observation in AAV-h $\alpha$-syn injected animals (Additional file 11: Figure S7). This result supports our hypothesis of a lack of sensitivity of SAMP8 and SAMR1 mice, mostly attributable to their AKR/J genetic background.

Taken together, our results in mice are three-fold. First, we established that overexpression of ha-syn induces neurodegeneration associated with accumulation 
a

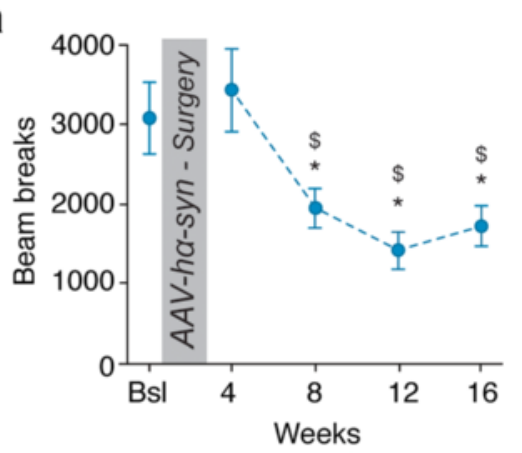

C Overground
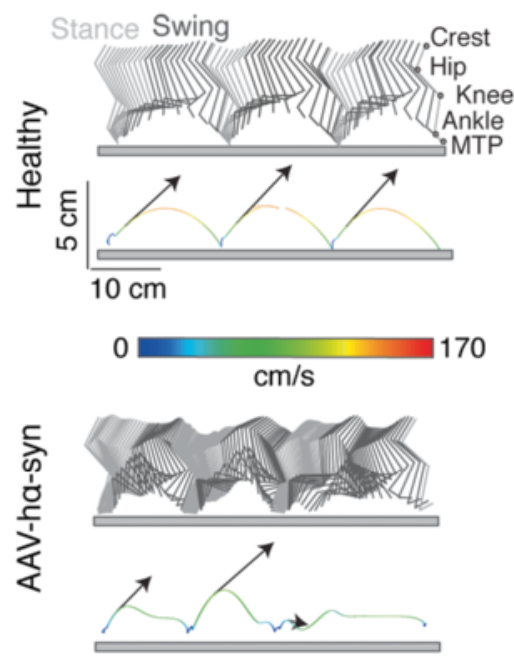

$\mathrm{n}=4$ Healthy 6 AAV-ha-syn

$\mathbf{f}$

Ladder

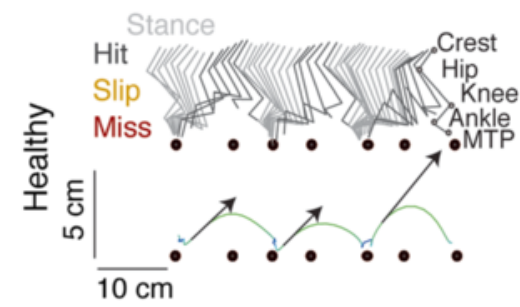

$0 \square \mathrm{cm} / \mathrm{s}$

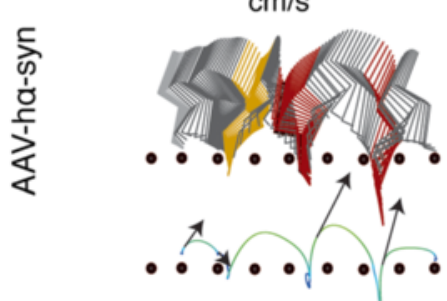

b

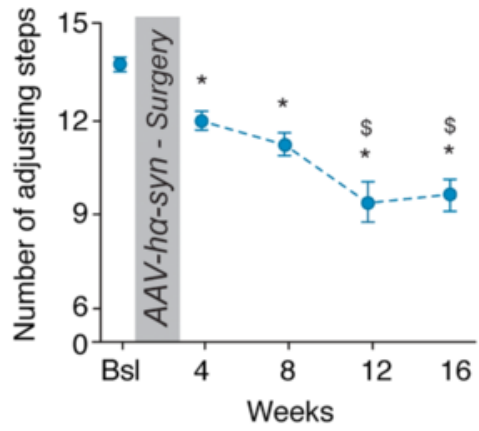

d Quantification of overground function

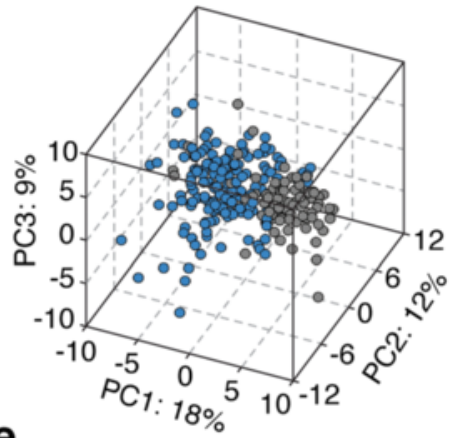

PC1 (18\%):

Parkinsonian gait

e

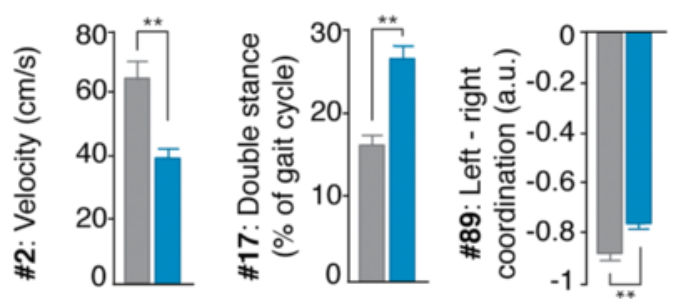

g

Quantification of ladder function
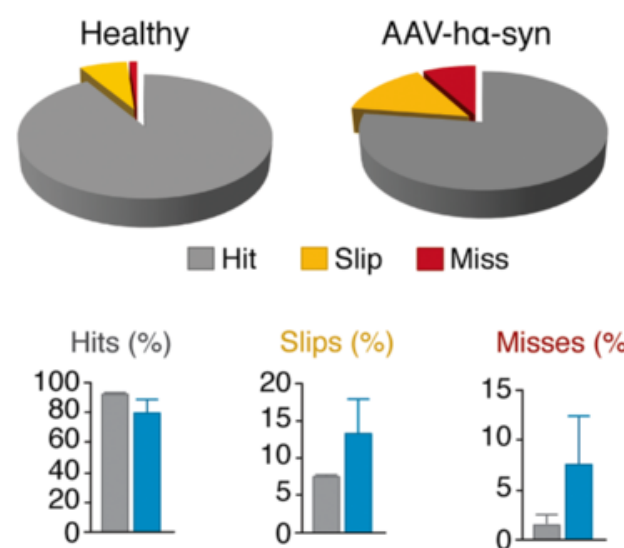

Misses (\%)

$n=3$ Healthy 5 AAV-ha-syn

Fig. 2 (See legend on next page.) 
(See figure on previous page.)

Fig. 2 Behavioral impairments associated with ha-syn overexpression in rats. a Spontaneous locomotor activity at baseline (Bsl) and at 4, 8, 12 and 16 weeks after surgery. b Stepping test at baseline and at 4,8,12 and 16 weeks after surgery. c Color-coded stick diagram decomposition of hindlimb movement for a representative healthy and AAV-ha-syn rat during crossing of an elevated horizontal ladder with irregularly spaced rungs (spacing $5.2+/-0.3 \mathrm{~cm}$ ). The corresponding endpoint trajectory is shown below. The colour code indicated the instantaneous velocity of the endpoint, while the arrows report the orientation and intensity of the foot push-off at swing onset. Light grey: stance; dark grey: hit; yellow: slip; dark red: miss. d Pie charts summarize total percentage of hits, slips, and misses (total of 182 steps evaluated, $\mathrm{n}$ are indicated in the legend boxes). Below percentage of hits, slips and misses are quantified as histogram plots. e The same representation is shown for a representative healthy and Parkinsonian rat during overground locomotion. f Principal component (PC) analysis was applied on 89 gait parameters measured during overground locomotion (10-15 gait cycles/hindlimb/rat, $n=4$ healthy and $n=6$ AAV-ha-syn rats). Each gait cycle is represented as a dots in the new space created by PC1-3. Histogram plot depicts mean values of PC1 scores for each experimental group. $\mathbf{g}$ Histogram plots represent mean values of parameters with high factor loading (|factor loading $>0.5$ ) on PC1, illustrating the most salient differences in motor control between the two groups. Numbers refer to Additional file 1: Table S1. ${ }^{*} p<0.05 ;{ }^{* *} p<0.01$; ${ }^{* * *} p<0.001$; $\$ p<0.05$ vs 4 weeks time point; error bars, SEM; PC, Principal Component; a.u., arbitrary unit

of $\alpha$-syn and p- $\alpha$-syn in mice. Second, phenotypic mutants with either accelerated senescence or resistance to senescence did not display differential susceptibility to ha-syn overexpression and MPTP intoxication. Third, in mice, p- $\alpha$-syn levels correlated with nigrostriatal degeneration.

\section{Ageing does not enhance susceptibility to a-syn overex- pression in marmoset monkeys}

Non-human primate neuronal physiology is closer to human than rodents. We thus took advantage of aged and young adult marmoset monkeys to decipher whether ageing might increase the susceptibility of dopamine neurons to degenerate following AAV-ho-syn injection.

The integrity of the nigrostriatal pathway was investigated in young adult (1-2 years old, $n=8)$ and old marmosets ( $>6$ years old, $n=5$ ). The life span is around 10 years, i.e., the old animals had exceeded their mid-life expectancy. In humans, PD typically occurs around 60 years of age. Sham-operated old animals displayed a significant reduction of $\mathrm{TH}$ immunoreactivity in the striatum (40\% in the caudate nucleus and $43 \%$ in the putamen) associated with a significant decrease of TH-positive cells in the SNpc (29\%) compared to sham-operated young animals (Fig. 4a). Animals were euthanized at 11 weeks post-surgery to avoid any losses due to death from natural causes of some old animals. AAV-h $\alpha$-syn induced a degeneration of TH-positive neurons of the same magnitude (13\% in young animals, $20 \%$ in old animals, $p=0.7182$ ). At the striatal level, h $\alpha$-syn overexpression produced depletion in striatal $\mathrm{TH}$-positive fibers in both caudate nucleus (8\% for young and $11 \%$ for old animals - Fig. 4a) and putamen (23\% for young animals and $15 \%$ for old animals - Fig. 4a). These results indicate that ho-syn-induced degeneration of the nigrostriatal pathway was not affected by the age of the animals.

We then investigated the occurrence and pattern of ha-syn pathology. The level of overexpression and pattern of ha-syn staining were identical at both SNpc and striatal levels in both groups (around $300 \%$ Fig. 4b). Then, we examined $\mathrm{p}-\alpha$-syn immunostaining in the SNpc (Fig. 4c). While both young and old animals showed p- $\alpha$-syn immunoreactivity only on the injected side, old animals exhibited a significantly lower staining than young animals (Fig. 4c). In contrast to mice (Fig. 3c) but in agreement with our results in rat (Additional file 8: Figure S4), p- $\alpha$-syn staining was mostly restricted to $\mathrm{SNpc}$ and could be found in neuronal perikarya and dendritic terminals (Fig. 4c). Nevertheless, p- $\alpha$-syn and the number of TH-positive neurons in the SNpc (Fig. 4d) did not covary, unlike in mice (Fig. 3d). Both age groups presented an identical level of nigrostriatal degeneration regardless of the $\mathrm{p}$ - $\alpha$-syn staining levels. This last observation raises questions as to the interpretation of $p-\alpha-$ syn levels in different species.

\section{Discussion}

Here we report that AAV2/9-mediated p.A53T mutated human $\alpha$-syn overexpression induces robust nigrostriatal neurodegeneration in mice, rats and monkeys, establishing translational comparisons among species. In addition, the comprehensive degeneration kinetics and high-resolution kinematic characterizations in the rat model uncovered clinically relevant relationships between the extent of the lesion and the behavioural PDlike phenotype.

Previous studies have shown that overexpression of human WT $\alpha$-syn in rats induces around $80 \%$ of dopamine neuron loss after 16 weeks [19], a result comparable to the present p.A53T mutant $\alpha$-syn overexpression in the rat ( $80 \%$ of DA cell loss after 16 weeks) and paralleled by only $30 \%$ dopamine neuron loss in mice after 20 weeks and $20 \%$ in aged marmoset monkeys after 11 weeks. Our results in the rat model are in agreement with previous reports $[10,13]$ using the same expression cassette, although the level and time frame of neurodegeneration varies depending on the titer and vector serotype used [10]. Patients harbouring p.A53T mutation do 


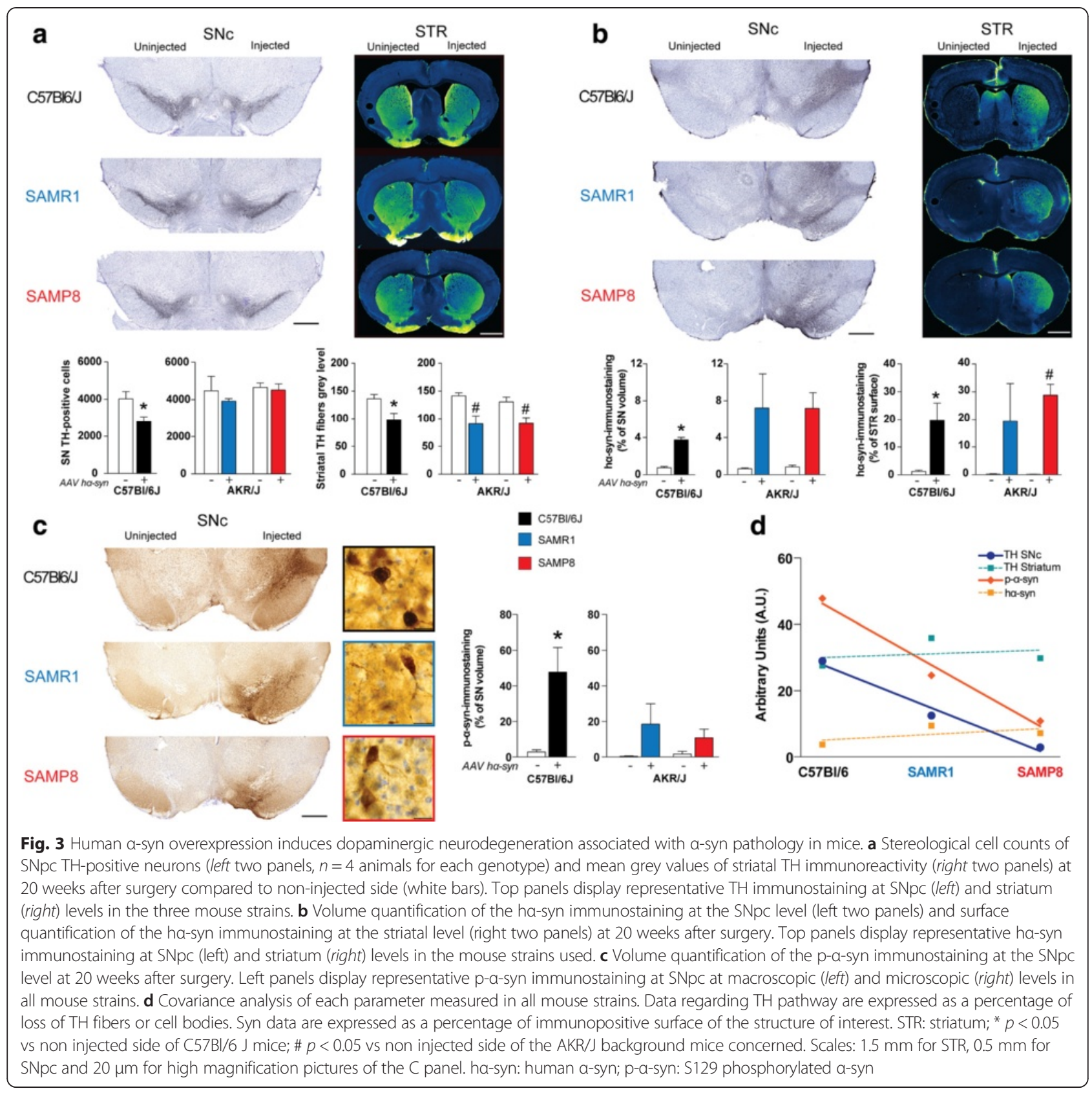

not present a classic parkinsonism but an early-onset, fast-progressing disease associated with secondary symptoms [26]. Of interest, the dynamics of WT $\alpha$-syn overexpression [19] fit with our degeneration curve (Fig. 1f) although one study reports a faster degeneration time course for p.A53T mutant $\alpha$-syn overexpression [20]. In mice, one study did not show any significant differences between WT and p.A53T mutated $\alpha$-syn overexpression [9]. Such differences might be attributable to the use of different expression cassettes, as it appears in other reports that dose of vector could be more important than the $\alpha$-syn mutation $[9,22]$. The present data suggest that this mutation might not have the same functional consequences in rodents and marmoset monkeys as in humans. The reason for the discrepancy between different clinical symptoms and identical rates of degeneration in preclinical models remains to be established. The presence of an alanine residue at position 53 is not fully conserved during evolution. Several species, including rodents and marmoset monkeys, exhibit a threonine instead of an alanine residue at position 53 in their endogenous $\alpha$-syn $[27,28]$. Investigation in a mammalian model that does not have the A53T substitution (such as macaques) might resolve this apparent discrepancy. 


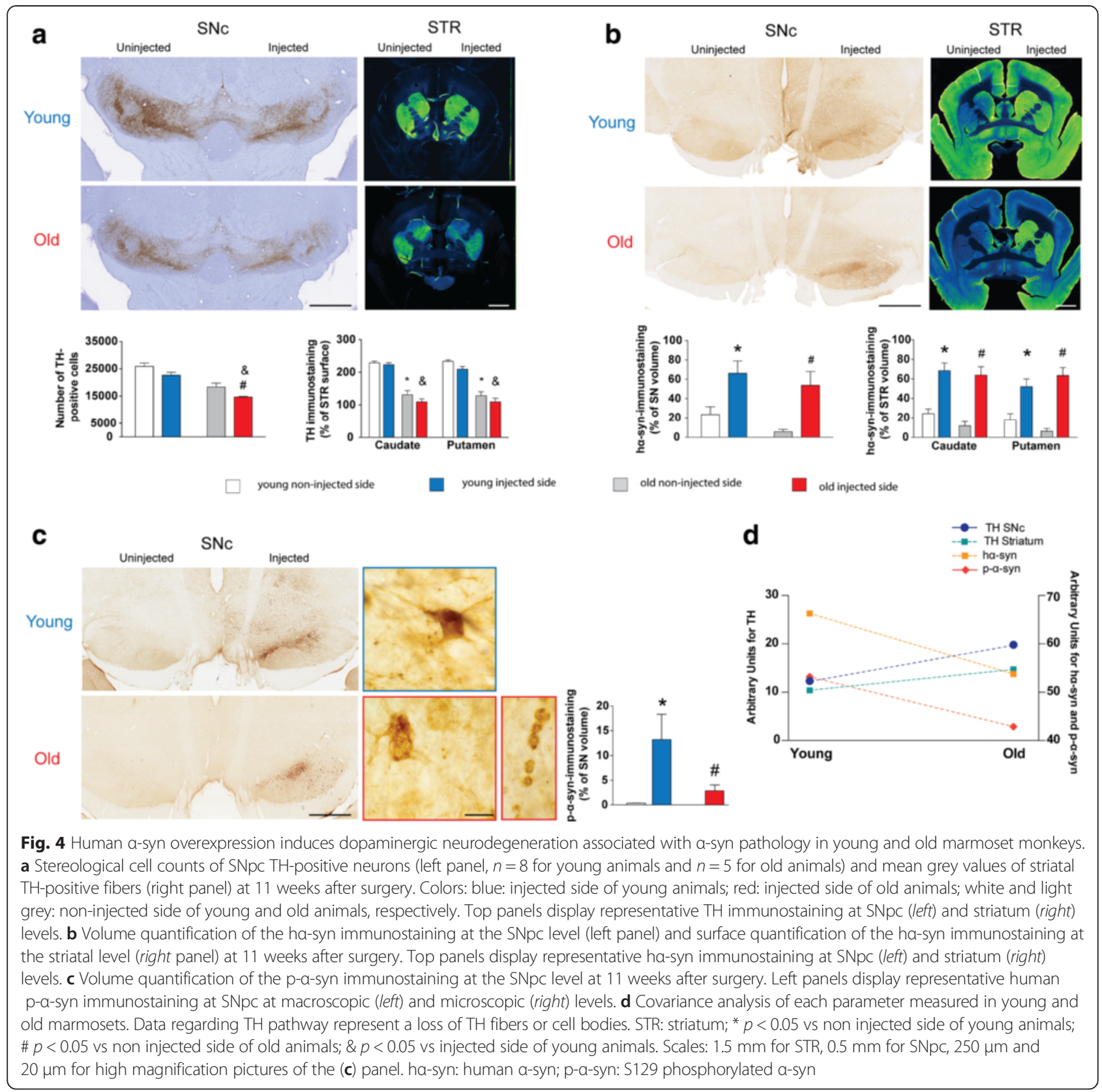

The behavioural phenotype of AAV-ho-syn-injected rats shares many features with the human disease. In the open field and locomotor tasks, AAV-ho-syn-injected rats displayed both akinesia and bradykinesia. L-3,4-dihydroxyphenylalanine treatment effectively alleviates these symptoms [15]. Here, we quantified these deficits using objective and quantitative analyses. We found that a decrease in velocity (gait hypokinesia) and abnormal gait, as evidenced by impaired inter-limb coordination and increased double stance were the main features altered in response to $\alpha$-syn over-expression. These features constitute the hallmarks of shuffling gait in PD patients [26]. The longitudinal follow-up of basic motor function allowed us to establish that motor dysfunction appears from 4 weeks onwards, when $60 \%$ of dopamine neurons and $80 \%$ of striatal dopaminergic fibers have been lost. These results are in perfect coherence with the known or hypothesized relationship between nigrostriatal lesions and symptoms in MPTP-treated macaques [29] and PD humans, respectively [30]. Applying the regression defined for the AAV-ho-syn-injected rats to the AAV-ho-syninjected mice and monkeys, for which we have a single 
time point, led us to predict that symptoms would be detected, post-surgery, at 133 weeks in mice, 244 weeks for young marmosets and 142 weeks for old marmosets. While such duration would exceed the mouse life span, rendering the model inadequate for behavioural studies, the durations are indeed compatible with marmoset life span. These extrapolations suggest that age may impact upon the development of symptoms, which remain to be confirmed. However, this long-term window excludes the involvement of marmoset monkeys in behavioural studies, restricting the use of this species for studies relying on post-mortem endpoints. Previous work suggested that, after the first occurrence of clinical signs, the evolution of the disease slows down [30]. Contrary to the MPTPtreated monkey model, in which degeneration of dopamine neurons fits with a linear regression [29, 30], AAV-h $\alpha$-syn injection in the rat followed an exponential regression equation characteristic of a fast onset of neurodegeneration followed by a slow evolution, a feature to bear in mind both for the study of the prodromal phase and for the validation of therapeutic approaches.

Several studies have reported the existence of $p$ - $\alpha$-syn in brains from PD patients [21]. $\alpha$-syn could indeed be phosphorylated at S129 in preclinical models of PD [19, 31, 32]. However, it remains unclear from in vitro and in vivo data whether phosphorylation of $\alpha$-syn impacts upon its fibrillation process [33]. We here show that mice exhibiting the highest $\mathrm{p}$ - $\alpha$-syn levels display the highest level of degeneration. However, the marmoset monkey data contradict this hypothesis as no correlation between the $\mathrm{p}$ - $\alpha$-syn levels and DA degeneration was found in this species. Several other reports have already questioned the relevance of $\mathrm{p}$ - $\alpha$-syn to the neurodegenerative process and as a therapeutic target [34]. Our data suggest that $p$ - $\alpha$-syn might have different impact depending upon the species, calling for caution in interpreting data obtained in rodents compared to primates. It may be noted that $\mathrm{p}-\alpha$-syn is found in healthy aged human brains [35].

A key objective of the present study was to address the role of ageing in the susceptibility of dopamine neurons to degeneration in mice and marmoset monkeys. In mice, aged animals are more prone to MPTP-induced striatal dopamine loss [36]. The absence of neurodegeneration after h $\alpha$-syn overexpression or MPTP administration observed in SAMP8 and SAMR1 mice might be due to their AKR/J background, which is known to be less sensitive to MPTP than $\mathrm{C} 57 \mathrm{Bl} / 6 \mathrm{~J}$ [22]. Whether this is indeed a lack of sensitivity or the consequence of an unknown neuroprotective factor remains to be established. Similar to the differential rat strain susceptibility, these results call for caution regarding genetic background when using genetically engineered or mutant mice for studying PD pathogenesis. A previous study using MPTP in marmoset monkeys reported that aged marmosets (8-10 years-old) were no more susceptible to MPTP intoxication than younger animals [37], a finding consistent with our results using $\alpha$-syn-induced neurodegeneration. In rhesus monkeys, aged animals (20-23 years-old) required a third as much MPTP to induce the same behavioural phenotype as in young animals [38], suggesting an age-dependent susceptibility. The differential MPTP susceptibility between these two species might be due to the presence of neuromelanin, a pigment that accumulates over time in the SNpc through oxidation of dopamine to aminochrome and polymerization, in macaques but not in marmosets [39]. Melanized neurons are more susceptible than non-melanized neurons both in PD patients [40] and MPTP-treated primates [41]. Further studies are needed to unravel whether the presence of neuromelanin in aged macaques, and to a lesser extent in young macaques, might impact the degeneration course following ho-syn overexpression. The specificity of primate brain for the accumulation of neuromelanin strengthens the special relevance of preclinical studies in primates for PD. Nevertheless, ageing here appears as a modest independent factor that only decreases the gap to a putative threshold for the appearance of PD symptoms in marmoset monkey. Our results thus strengthen the multiple-hit hypothesis [4, 42]. We propose that a combination of deleterious events accelerate the ageingrelated decline in dopamine neuron function, rather than the stochastic acceleration hypothesis in which ageing directly sensitizes the dopamine system [3].

\section{Conclusions}

Ageing might only be a contributing factor to the extent of ho-syn-induced degeneration, suggesting that mature adult animals might be enough for testing diseasemodifying therapies. Since PD patients would only benefit from such therapy after symptom onset, i.e., when they already have a loss of $70-80 \%$ of striatal dopaminergic terminals and 50-60\% of dopamine neurons, the present study defines the most suitable time points for therapeutic intervention in those three models. Considering the identified dynamics (Fig. 1), we propose that starting administration from 3-4 weeks after surgery in the AAV-ho-syn rat model would be most clinicallyrelevant, offering translatable behavioural and postmortem endpoints while the mouse and marmoset models should be used for post-mortem endpoints only.

\section{Availability of supporting data}

The data sets supporting the results of this article are included within the article and its additional files. 


\section{Additional files.}

\section{Additional file 1: Table S1. Computed kinematic and kinematic} variables.

Additional file 2: Figure S6. Extraction of gait parameters affected by ha-syn overexpression in rats. PC analysis was applied on all gait parameters measured during overground locomotion, as reported in Fig. 2. Parameters correlating with PC1 (|loading factor $\mid>0.5$ ) and thus specifically altered by ha-syn overexpression were regrouped into functional clusters, which we named for clarity. The numbers refer to Table S1. Some of the parameters with high factor loadings are reported in the histogram plots of Fig. 2.

Additional file 3: Table S2. List of antibodies used in the study Additional file 4: Table S3. Sampling and size of probes used in the study.

Additional file 5: Figure S1. rAAV2/9 vector-mediated overexpression of ha-syn in rat SNpc induces progressive dopaminergic neurodegeneration related to ha-syn expression dynamics. (a-e) Histogram plots represent mean values of DA related $(\mathrm{a}-\mathrm{c})$ or ha-syn $(\mathrm{d}, \mathrm{e})$ related markers. ${ }^{*}: p<0.05$ vs sham animals, $\$: p<0.05$ vs $72 \mathrm{~h}, \#: p<0.05$ vs 1 week. Errors bars are SEM. ha-syn: human a-syn.

Additional file 6: Figure S2. Striatal and SNpc ha-syn expression. Delay between SNpc (a) and striatal (b) ha-syn expression. Ha-syn pattern in the SNpc starting from a strong cellular staining (a - early time points) to a more diffuse one (a - late timepoints). In the striatum, morphological change of ha-syn staining from a long-wire staining (b - early timepoints) to a more punctiform one (b - late time points). Scale bars: (a) $50 \mu \mathrm{m}$, (b) 20 นm. ha-syn: human a-syn.

Additional file 7: Figure S3. Presence of ubiquitin-ha-syn colocalization in SNpc remaining neurons. (a,e,i) TH staining, $(b, f, j)$ ubiquitin, $(c, g, k)$ ha-syn $(\mathrm{d}, \mathrm{h}, \mathrm{l})$ merge. $(\mathrm{a}-\mathrm{h}) \times 10$ magnification, $(\mathrm{i}-\mathrm{l}) \times 40$ magnification. ha-syn: human a-syn

Additional file 8: Figure S4. $p-a-s y n$ staining in the SNpc. (a-d) Representative photomicrographs of p-a-syn staining in the SNpc of sham (a) or injected (b-d) animals. In contrast to ha-syn staining, p-a-syn is highly localized in neuronal perikarya (c) and neurites (d) at 16 weeks after surgery. (e) Quantification of the SNpc volume positive for p-a-syn staining. Scale bar: (a,b) $1 \mathrm{~mm}$, (c) $20 \mu \mathrm{m}$. ha-syn: human a-syn, pa-syn: S129 phosphorylated a-syn.

Additional file 9: Figure S5. Wistar rats are significantly less sensitive to ha-syn overexpression than Sprague Dawley rats. Histogram plots represent number of TH-positive cells in the SNpc at 8 weeks after surgery for sham (white bars) and AAV-ha-syn injected rats (black bars). ***: $p<0.01$ vs control side, \#: $p<0.05$. Error bars: SEM.

Additional file 10: Movie 1. Locomotion across an horizontal ladder in sham and AAV-ha-syn-injected rats. This movie shows a representative kinematic recording of quadrupedal locomotion along the equally spaced rungs of an horizontal ladder in a sham operated and an

AAV-ha-syn-injected rat.

Additional file 11: Figure S7. SAMP8 and SAMR1 mice do not present nigrostriatal degeneration after MPTP intoxication while C57BI/6 J does. Histogram plots represent the number of remaining TH-positive cells in the SNpc 21 days after the last injection of MPTP. * : $p<0.05$ vs non injected animals.

\section{Abbreviations}

a-syn: Alpha-synuclein; AAV: Recombinant adeno-associated virus; DA: Dopamine; DAT: Dopamine transporter; ha-syn: Human mutant p.A53T alpha-synuclein; LB: Lewy bodies; LN: Lewy neurites; MPTP: 1-methyl-1,2,3,6-tetrahydropyridine; PC: Principal component; PD: Parkinson's disease; SAMP8: Senescence-accelerated mouse-prone 8; SAMR1: Senescence-accelerated mouse-resistant 1; SNpc: Substantia nigra pars compacta; TH: Tyrosine Hydroxylase; WRPE: Woodchuck hepatitis virus post-transcriptional regulatory element; WT: Wild-type.

\section{Competing interests}

The authors declare that they have no competing interests.

\section{Authors' contributions}

G.C., E.B., and B.D. designed research; M.Bo., S.D., M.E., S.B., M.Ba., I.V., L.B., C.P., V.G., T.B., G.P., Q.L., P.O.F., F.G., G.C. and B.D. performed research; N.D., V.B., D.S., and A.M. contributed new reagents/analytic tools; M.Bo., S.D., B.D. and E.B. analyzed data; and M.Bo., P.O.F., G.C., E.B., and B.D. wrote the paper. All authors read and approved the final manuscript.

\section{Acknowledgments}

Our warm thanks to Pr. Alan R. Crossman for editing the manuscript. The University of Bordeaux and the Centre National de la Recherche Scientifique provided infrastructural support. This work was supported by Agence Nationale de la Recherche grants (ANR-12-BSV4-0001-01, LABEX BRAIN ANR-10-LABX-43), Fondation de France, and European Union 7th Generation Framework grant NeuWalk (CP-IP 258654). Mathieu Bourdenx is a recipient of an MESR fellowship. Slide scanning was performed at the Bordeaux Imaging Center a service unit of the CNRS-INSERM and Bordeaux University, member of the national infrastructure France Biolmaging. Accommodations and experiments were performed in the: Service des Animaleries of the University of Bordeaux, supported by the Région Aquitaine. The help of Elodie Poinama and Laetitia Medan is acknowledged. We thank Marie-Laure Thiolat and Li Hao for their excellent technical support.

\section{Author details}

${ }^{1}$ University de Bordeaux, Institut des Maladies Neurodégénératives, UMR 5293, 33000 Bordeaux, France. ${ }^{2}$ CNRS, Institut des Maladies Neurodégénératives, UMR 5293, 33000 Bordeaux, France. ${ }^{3}$ International Paraplegic Foundation chair in spinal cord Repair, Center for Neuroprosthetics and Brain Mind Institute, School of Life Sciences, Swiss Federal Institute of Technology (EPFL), Lausanne, Switzerland. ${ }^{4}$ Motac Neuroscience, Manchester, UK. ${ }^{5}$ Institute of Laboratory Animal Sciences, China Academy of Medical Sciences, Beijing, China. ${ }^{6}$ Department of Neurosciences and Leuven Research Institute for Neuroscience and Disease (LIND), KU Leuven, Laboratory for Neurobiology and Gene Therapy, Kapucijnenvoer 33, box 7001, 3000 Leuven, Belgium. 'Parkinson's Disease Pharmacology, UCB Pharma S.A., Braine L'Alleud B-1420, Belgium. ${ }^{8}$ University de Bordeaux, Interdisciplinary Institute for Neuroscience, UMR5297, Bordeaux, France. ${ }^{9}$ CNRS, Institut Interdisciplinaire de Neurosciences, UMR 5297, F-33000 Bordeaux, France.

Received: 29 June 2015 Accepted: 6 July 2015

Published online: 25 July 2015

\section{References}

1. Dehay B, Bourdenx M, Gorry P, Przedborski S, Vila M, Hunot S, Singleton A, Olanow CW, Merchant KM, Bezard E, Petsko GA, Meissner WG (2015) Targeting alpha-synuclein for treatment of Parkinson's disease: mechanistic and therapeutic considerations. Lancet Neurol. doi:10.1016/S1474-4422(15)00006-X

2. Mattson MP, Magnus T (2006) Ageing and neuronal vulnerability. Nat Rev Neurol 7(4):278-294. doi:10.1038/nrn1886

3. Collier TJ, Kanaan NM, Kordower JH (2011) Ageing as a primary risk factor for Parkinson's disease: evidence from studies of non-human primates. Nat Rev Neurol 12(6):359-366. doi:10.1038/nrn3039

4. Sulzer D (2007) Multiple hit hypotheses for dopamine neuron loss in Parkinson's disease. Trends Neurosci 30(5):244-250. doi:10.1016/ j.tins.2007.03.009

5. Spillantini MG, Schmidt ML, Lee VM, Trojanowski JQ, Jakes R, Goedert M (1997) Alpha-synuclein in Lewy bodies. Nature 388(6645):839-840. doi:10.1038/42166

6. Polymeropoulos MH, Lavedan C, Leroy E, Ide SE, Dehejia A, Dutra A, Pike B, Root H, Rubenstein J, Boyer R, Stenroos ES, Chandrasekharappa S, Athanassiadou A, Papapetropoulos T, Johnson WG, Lazzarini AM, Duvoisin RC, Di lorio G, Golbe LI, Nussbaum RL (1997) Mutation in the alphasynuclein gene identified in families with Parkinson's disease. Science 276(5321):2045-2047

7. Magen I, Chesselet MF (2010) Genetic mouse models of Parkinson's disease The state of the art. Prog Brain Res 184:53-87. doi:10.1016/ S0079-6123(10)84004-X

8. Bezard E, Yue Z, Kirik D, Spillantini MG (2013) Animal models of Parkinson's disease: limits and relevance to neuroprotection studies. Mov Disord: Off J Mov Dis Soc 28(1):61-70. doi:10.1002/mds.25108 
9. Oliveras-Salva M, Van der Perren A, Casadei N, Stroobants S, Nuber S, D'Hooge R, Van den Haute C, Baekelandt V (2013) rAAV2/7 vector-mediated overexpression of alpha-synuclein in mouse substantia nigra induces protein aggregation and progressive dose-dependent neurodegeneration. Mol Neurodegener 8:44. doi:10.1186/1750-1326-8-44

10. Van der Perren A, Toelen J, Casteels C, Macchi F, Van Rompuy AS, Sarre S, Casadei N, Nuber S, Himmelreich U, Osorio Garcia MI, Michotte Y, D'Hooge R, Bormans G, Van Laere K, Gijsbers R, Van den Haute C, Debyser Z, Baekelandt V (2015) Longitudinal follow-up and characterization of a robust rat model for Parkinson's disease based on overexpression of alpha-synuclein with adeno-associated viral vectors. Neurobiol Aging 36(3):1543-1558. doi:10.1016/ j.neurobiolaging.2014.11.015

11. Zolotukhin S, Byrne BJ, Mason E, Zolotukhin I, Potter M, Chesnut K, Summerford C, Samulski RJ, Muzyczka N (1999) Recombinant adeno-associated virus purification using novel methods improves infectious titer and yield. Gene Ther 6(6):973-985

12. Aurnhammer C, Haase M, Muether N, Hausl M, Rauschhuber C, Huber I, Nitschko H, Busch U, Sing A, Ehrhardt A, Baiker A (2012) Universal real-time PCR for the detection and quantification of adenoassociated virus serotype 2-derived inverted terminal repeat sequences. Hum Gene Ther Methods 23(1):18-28. doi:10.1089/ hgtb.2011.034

13. Engeln M, Fasano S, Ahmed SH, Cador M, Baekelandt V, Bezard E, Fernagut PO (2013) Levodopa gains psychostimulant-like properties after nigral dopaminergic loss. Ann Neurol 74(1):140-144. doi:10.1002/ana.23881

14. Dominici N, Keller U, Vallery H, Friedli L, van den Brand R, Starkey ML, Musienko P, Riener R, Courtine G (2012) Versatile robotic interface to evaluate, enable and train locomotion and balance after neuromotor disorders. Nat Med 18(7):1142-1147. doi:10.1038/nm.2845

15. Engeln M, Ahmed SH, Vouillac C, Tison F, Bezard E, Fernagut PO (2013) Reinforcing properties of Pramipexole in normal and parkinsonian rats. Neurobiol Dis 49:79-86. doi:10.1016/j.nbd.2012.08.005

16. Engeln M, Bastide MF, Toulme E, Dehay B, Bourdenx M, Doudnikoff $E_{\text {, }}$ Li Q, Gross CE, Boue-Grabot E, Pisani A, Bezard E, Fernagut PO (2014) Selective Inactivation of Striatal FosB/DeltaFosB-Expressing Neurons Alleviates L-Dopa-Induced Dyskinesia. Biol Psychiatry. doi:10.1016/ j.biopsych.2014.07.007

17. van den Brand R, Heutschi J, Barraud Q, DiGiovanna J, Bartholdi K, Huerlimann M, Friedli L, Vollenweider I, Moraud EM, Duis S, Dominici N, Micera S, Musienko P, Courtine G (2012) Restoring voluntary control of locomotion after paralyzing spinal cord injury. Science 336(6085): 1182-1185. doi:10.1126/science.1217416

18. Vitrac C, Peron S, Frappe I, Fernagut PO, Jaber M, Gaillard A, Benoit-Marand M (2014) Dopamine control of pyramidal neuron activity in the primary motor cortex via D2 receptors. Frontiers Neural Circuits 8:13. doi:10.3389/ fncir.2014.00013

19. Decressac M, Mattsson B, Lundblad M, Weikop P, Bjorklund A (2012) Progressive neurodegenerative and behavioural changes induced by AAV-mediated overexpression of alpha-synuclein in midbrain dopamine neurons. Neurobiol Dis 45(3):939-953. doi:10.1016/ j.nbd.2011.12.013

20. Kirik D, Rosenblad C, Burger C, Lundberg C, Johansen TE, Muzyczka N, Mandel RJ, Bjorklund A (2002) Parkinson-like neurodegeneration induced by targeted overexpression of alpha-synuclein in the nigrostriatal system. J Neurosci 22(7):2780-2791

21. Fujiwara H, Hasegawa M, Dohmae N, Kawashima A, Masliah E, Goldberg MS, Shen J, Takio K, Iwatsubo T (2002) alpha-Synuclein is phosphorylated in synucleinopathy lesions. Nat Cell Biol 4(2):160-164. doi:10.1038/ncb748

22. Hamre K, Tharp R, Poon K, Xiong X, Smeyne RJ (1999) Differential strain susceptibility following 1-methyl-4-phenyl-1,2,3,6-tetrahydropyridine (MPTP) administration acts in an autosomal dominant fashion: quantitative analysis in seven strains of Mus musculus. Brain Res 828(1-2):91-103

23. Takeoka A, Vollenweider I, Courtine G, Arber S (2014) Muscle spindle feedback directs locomotor recovery and circuit reorganization after spinal cord injury. Cell 159(7):1626-1639. doi:10.1016/..cell.2014.11.019

24. Takeda T, Hosokawa M, Higuchi K, Hosono M, Akiguchi I, Katoh H (1994) A novel murine model of aging, Senescence-Accelerated Mouse (SAM). Arch Gerontol Geriatr 19(2):185-192

25. Jackson-Lewis V, Przedborski S (2007) Protocol for the MPTP mouse model of Parkinson's disease. Nat Protoc 2(1):141-151. doi:10.1038/nprot.2006.342
26. Dehay B, Bourdenx M, Gorry P, Przedborski S, Vila M, Hunot S, Singleton A, Olanow CW, Merchant KM, Bezard E, Petsko GA, Meissner WG (2015) Targeting a-synuclein for treating Parkinson's disease: mechanistic and therapeutic considerations. Lancet Neurology. doi:10.1016/S1474-4422(15)00006-X

27. Larsen K, Hedegaard C, Bertelsen MF, Bendixen C (2009) Threonine 53 in alpha-synuclein is conserved in long-living non-primate animals. Biochem Biophys Res Commun 387(3):602-605. doi:10.1016/ j.bbrc.2009.07.070

28. Hamilton BA (2004) alpha-Synuclein A53T substitution associated with Parkinson disease also marks the divergence of Old World and New World primates. Genomics 83(4):739-742. doi:10.1016/j.ygeno.2003.09.016

29. Bezard E, Dovero S, Prunier C, Ravenscroft P, Chalon S, Guilloteau D, Crossman AR, Bioulac B, Brotchie JM, Gross CE (2001) Relationship between the appearance of symptoms and the level of nigrostriatal degeneration in a progressive 1-methyl-4-phenyl-1,2,3,6-tetrahydropyridine-lesioned macaque model of Parkinson's disease. J Neurosci 21(17):6853-6861

30. Bezard E, Gross CE (1998) Compensatory mechanisms in experimental and human parkinsonism: towards a dynamic approach. Prog Neurobiol 55(2):93-116

31. Eslamboli A, Romero-Ramos M, Burger C, Bjorklund T, Muzyczka N, Mandel RJ, Baker H, Ridley RM, Kirik D (2007) Long-term consequences of human alpha-synuclein overexpression in the primate ventral midbrain. Brain :J Neuro 130(Pt 3):799-815. doi:10.1093/brain/awl382

32. Lo Bianco C, Ridet IL, Schneider BL, Deglon N, Aebischer P (2002) alpha -Synucleinopathy and selective dopaminergic neuron loss in a rat lentiviral-based model of Parkinson's disease. Proc Natl Acad Sci U S A 99(16):10813-10818. doi:10.1073/pnas.152339799

33. Paleologou KE, Schmid AW, Rospigliosi CC, Kim HY, Lamberto GR, Fredenburg RA, Lansbury PT Jr, Fernandez CO, Eliezer D, Zweckstetter M, Lashuel HA (2008) Phosphorylation at Ser-129 but not the phosphomimics S129E/D inhibits the fibrillation of alpha-synuclein. $J$ Biol Chem 283(24):16895-16905. doi:10.1074/jbc.M800747200

34. Buck K, Landeck N, Ulusoy A, Majbour NK, El-Agnaf OM, Kirik D (2015) Ser129 phosphorylation of endogenous alpha-synuclein induced by overexpression of polo-like kinases 2 and 3 in nigral dopamine neurons is not detrimental to their survival and function. Neurobiol Dis 78:100-114. doi:10.1016/ j.nbd.2015.03.008

35. Muntane G, Ferrer I, Martinez-Vicente M (2012) alpha-synuclein phosphorylation and truncation are normal events in the adult human brain. Neuroscience 200:106-119. doi:10.1016/ j.neuroscience.2011.10.042

36. Irwin I, Finnegan KT, Delanney LE, Di Monte D, Langston JW (1992) The relationships between aging, monoamine oxidase, striatal dopamine and the effects of MPTP in C57BL/6 mice: a critical reassessment. Brain Res 572(1-2):224-231

37. Rose $S$, Nomoto $M$, Jackson EA, Gibb WR, Jaehnig $P$, Jenner $P$, Marsden CD (1993) Age-related effects of 1-methyl-4-phenyl-1,2,3,6-tetrahydropyridine treatment of common marmosets. Eur J Pharmacol 230(2):177-185

38. Ovadia A, Zhang Z, Gash DM (1995) Increased susceptibility to MPTP toxicity in middle-aged rhesus monkeys. Neurobiol Aging 16(6):931-937

39. Paris I, Lozano J, Perez-Pastene C, Munoz P, Segura-Aguilar J (2009) Molecular and neurochemical mechanisms in PD pathogenesis. Neurotox Res 16(3):271-279. doi:10.1007/s12640-009-9059-4

40. Hirsch E, Graybiel AM, Agid YA (1988) Melanized dopaminergic neurons are differentially susceptible to degeneration in Parkinson's disease. Nature 334(6180):345-348. doi:10.1038/334345a0

41. Herrero MT, Hirsch EC, Kastner A, Ruberg M, Luquin MR, Laguna J, Javoy-Agid F, Obeso JA, Agid Y (1993) Does neuromelanin contribute to the vulnerability of catecholaminergic neurons in monkeys intoxicated with MPTP? Neuroscience 56(2):499-511

42. Carvey PM, Punati A, Newman MB (2006) Progressive dopamine neuron loss in Parkinson's disease: the multiple hit hypothesis. Cell Transplant 15(3):239-250 